\title{
Influence effect of an external electric field and dissipative tunneling on intracenter optical transitions in quantum molecules with $D_{2}^{-}$states
}

\author{
V. D. Krevchik ${ }^{1}$, A. V. Razumov ${ }^{1}$, P. V. Krevchik ${ }^{1}$, M. B. Semenov ${ }^{1}$, A. V. Shorokhov ${ }^{1,2,3,4}$, \\ A. P. Shkurinov ${ }^{5,1}$, I. A. Ozheredov ${ }^{5,1}$, Y.H. Wang 6 , T. R. Li ${ }^{6}$, A. K. Malik \\ ${ }^{1}$ Penza State University, Krasnaya str., 40, Penza, 440026, Russia \\ ${ }^{2}$ University of Jyväskylä, P.O. Box 35 (YFL), Jyväskylä, 40014, Finland \\ ${ }^{3}$ National Research Mordovia State University, Saransk, 430005, Russia \\ ${ }^{4}$ International Research Centre Mag Top, Institute of Physics, Polish Academy of Sciences, \\ Aleja Lotnikow 32/46, Warsaw, PL-02668, Poland \\ ${ }^{5}$ Institute for Problems of Laser and Information Technologies RAS, Moscow reg., 140700, Russia \\ ${ }^{6}$ Lanzhou University, Lanzhou, 730000, China \\ ${ }^{7}$ Multanimal Modi College Modinagar, Uttar Prasesh, 201204, India \\ Misha29.02.1@gmail.com
}

\section{PACS 73.40.Gk, 03.65.Xp}

DOI 10.17586/2220-8054-2020-11-5-510-518

\begin{abstract}
In the zero-range potential model and in the effective mass approximation, dispersion equations have been obtained, that describe dependence of the average binding energies of the quasistationary g- and u-states of the $D_{2}^{-}$- center in the $\mathrm{QD}$, as well as the widths of energy levels on the magnitude of the external electric field and the parameters of 1D-dissipative tunneling. Dips in the field dependences of the binding energies average values for quasi-stationary $\mathrm{g}$ - and $\mathrm{u}$-states have been revealed. It is shown that the field dependences of the energy level widths for the $\mathrm{g}$ - and $\mathrm{u}$ - states of the $D_{2}^{-}$-center have a resonance structure at the external electric field strengths corresponding to the dips in the field dependences of the average binding energies.

In the dipole approximation, the field dependence of the probability of the electron radiative transition from a quasistationary u-state to a quasi-stationary g-state of the $D_{2}^{-}$-center in a QD in the presence of dissipative tunneling with the participation of two local phonon modes has been calculated. It was found that the curve of the radiative transition probability (RTP) dependence on the strength of the external electric field contains three peaks.
\end{abstract}

Keywords: dissipative tunneling, quantum molecules, optical transitions.

Received: 4 August 2020

Revised: 1 September 2020

Final revision: 7 September 2020

\section{Introduction}

In recent years there has been an increasing interest in the optical properties of tunnel-coupled semiconductor nanostructures with impurity quasistationary states (a review is given in [1]). This interest is twofold, since, on the one hand, tunneling structures with impurity states are attractive from the point of view of creating new sources of stimulated emission based on intracenter optical transitions, and the further development of optoelectronics requires the search for effective methods for controlling the lifetime of impurity states. On the other hand, the combination of optical and tunneling measurements can serve as an important tool for investigating new effects associated with electron - phonon interactions and interparticle correlations in low-dimensional systems.

The problem of tunneling decay of quasistationary states in mesosystems of different nature (in various problems of physics, chemistry, and biology) is the subject of many monographs, reviews, and articles (see, for example [2-12]). Typical shapes of potential energy surfaces are quite universal in various applications. The problem of controlled tunnel transport in low-dimensional systems is relevant and is represented by a fairly wide range of experimental works [7-13]. Currently, an alternative to quantum methods for calculating the tunneling probability can be the instanton method proposed by A. M. Polyakov [14] and S. Coleman [15](a review is given in [2,3]), which allows one to take into account the influence of the heat bath on the tunneling transfer process. The theory of dissipative quantum tunneling as applied to systems with Josephson contacts was developed by E. J. Legget, A. I. Larkin, Yu. N. Ovchinnikov and others [2,3]. In the works of V. A. Bendersky, E. V. Vetoshkin and E. I. Kats (see, for example [16]) on basis of the instanton approach, E. Kats developed a quasi-classically exact method that makes it possible to solve the problem of tunneling splitting for symmetric double-well potentials in a wide energy range from the ground state to states located near the top of the barrier. The instanton method turned out to be productive in calculating the tunneling probability for QMs with $H^{-}$-like quasistationary impurity states [17], where, in combination with the zero-range 
potential method, it was possible to obtain the main results in an analytical form and to analyze the effect of tunneling decay on the optical properties of QDs. The need to take into account the QM interaction in a quasi-zero-dimensional structure, as well as the influence of local phonon modes on the field dependence of the probability of dissipative tunneling, requires further development of the instanton method as applied to the optics of low-dimensional tunneling structures with impurity quasi-stationary states.

The aim of this work is to study theoretically the features of intracenter radiative transitions in quantum molecules with quasi-stationary impurity $D_{2}^{-}$-states associated with the presence of $1 \mathrm{D}$-dissipative tunneling, taking into account the influence of two local phonon modes in an external electric field.

\section{Model: Dispersion equations describing quasi-stationary states of the $D_{2}^{-}$-center in a quantum dot in the presence of an external electric field and dissipative tunneling, taking into account the influence of two local phonon modes.}

Let us consider the problem of bound states of an electron localized at a $D_{2}^{-}$-center with quasistationary g- and $\mathrm{u}$-states in a QD with a parabolic confinement potential in the presence of an external electric field.

Let $D^{0}$ are the centers of the ion, which are localized at points with coordinates $R_{a 1}=\left(x_{a 1}, y_{a 1}, z_{a 1}\right)$ and $R_{a 2}=\left(x_{a 2}, y_{a 2}, z_{a 2}\right)$, here $R_{a i}=\left(x_{a i}, y_{a i}, z_{a i}\right)(i=1,2)$ - rectangular Cartesian coordinates of $D^{0}$-centers in QD. Let us assume that the external electric field $E_{0}$ is directed along the $x$ coordinate axis.

The two-center point perturbation potential $V_{\delta}$ can be written correctly in the form of a pseudopotential [18] as:

$$
V_{\delta}\left(\mathbf{r} ; \mathbf{R}_{a 1}, \mathbf{R}_{a 2}\right)=\sum_{i=1}^{2} \alpha_{i} \delta\left(\mathbf{r}-\mathbf{R}_{a i}\right)\left[1+\left(\mathbf{r}-\mathbf{R}_{a i}\right) \nabla_{\mathbf{r}}\right]
$$

Here, $\alpha_{i}$ is determined by the energy $E_{i}=-\hbar^{2} \alpha_{i}^{2} /\left(2 m^{*}\right)$ of the electronic bound state at the same $D^{0}$-centers in the bulk semiconductor; $m^{*}$ is the effective mass of an electron.

For one-electron states, unperturbed by impurities in a longitudinal electric field, the Hamiltonian in the chosen model of the parabolic confinement potential has the form:

$$
\mathbf{H}=-\frac{\hbar^{2}}{\left(2 m^{*}\right)} \nabla^{2}+\frac{1}{2} m^{*} \omega_{0}^{2}\left(x^{2}+y^{2}+z^{2}\right)-|e| E_{0} x,
$$

where $\omega_{0}$ - characteristic frequency of the QD confinement potential; $|e|$ - absolute value of the electron charge.

The eigenvalues $E_{n 1, n 2, n 3}$ and the corresponding eigenfunctions $\Psi_{n 1, n 2, n 3}(x, y, z)$ of the Hamiltonian (2) are given by expressions of the form [19]:

$$
\begin{gathered}
E_{n_{1}, n_{2}, n_{3}}=\hbar \omega_{0}\left(n_{1}+n_{2}+n_{3}+\frac{3}{2}\right)-\frac{|e|^{2} E_{0}^{2}}{2 m^{*} \omega_{0}^{2}}, \\
\Psi_{n_{1}, n_{2}, n_{3}}(x, y, z)=2^{-\frac{n_{1}+n_{2}+n_{3}}{2}}\left(n_{1} ! n_{2} ! n_{3} !\right)^{-\frac{1}{2}} \pi^{-\frac{3}{4}} a^{-\frac{3}{2}} \exp \left(-\left[\left(x-x_{0}\right)^{2}+y^{2}+z^{2}\right] /\left(2 a^{2}\right)\right) \times
\end{gathered}
$$

where $n_{1}, n_{2}, n_{3}=0,1,2, \ldots$ are the quantum numbers corresponding to the energy levels of an oscillatory spherically symmetric potential well; $a=\sqrt{\hbar /\left(m^{*} \omega_{0}\right)}$ is the characteristic length of the confinement potential; $x_{0}=$ $|e| E_{0} /\left(m^{*} \omega_{0}^{2}\right) ; H_{n}(x)$ are the Hermite polynomials.

In the effective mass approximation, the wave function of an electron $\Psi_{\lambda}\left(r ; R_{a 1}, R_{a 2}\right)$, localized at the $D_{2}^{-}$-center, satisfies the Lippmann-Schwinger equation:

$$
\Psi_{\lambda}\left(\mathbf{r} ; \mathbf{R}_{a 1}, \mathbf{R}_{a 2}\right)=\int d \mathbf{r}_{1} G\left(\mathbf{r}, \mathbf{r}_{1} ; E_{\lambda}\right) V_{\delta}\left(\mathbf{r}_{1} ; \mathbf{R}_{a 1}, \mathbf{R}_{a 2}\right) \Psi_{\lambda}\left(\mathbf{r}_{1} ; \mathbf{R}_{a 1}, \mathbf{R}_{a 2}\right)
$$

and has the form of a linear combination

$$
\Psi_{\lambda}\left(r ; R_{a 1}, R_{a 2}\right)=\sum_{k=1}^{2} \gamma_{k} c_{k} G\left(r ; R_{a k} ; E_{\lambda}\right),
$$

where $c_{k}=\lim _{\mathbf{r} \rightarrow \mathbf{R}_{a k}}\left[1+\left(\mathbf{r}-\mathbf{R}_{a k}\right) \nabla_{r}\right] \Psi_{\lambda}(k=1,2) ; G\left(\mathbf{r}, \mathbf{R}_{a k} ; E_{\lambda}\right)$ is the one-electron Green's function corresponding to a source at the point $\mathbf{R}_{a i}$ and the complex energy $E_{\lambda}=\hbar^{2} \lambda^{2} /\left(2 m^{*}\right)$.

The one-electron Green's function has the form: 


$$
G\left(\mathbf{r}, \mathbf{r}_{1} ; E_{\lambda}\right)=\sum_{n_{1}, n_{2}, n_{3}} \frac{\Psi_{n_{1}, n_{2}, n_{3}}^{*}\left(\mathbf{r}_{1}\right) \Psi_{n_{1}, n_{2}, n_{3}}(\mathbf{r})}{E_{\lambda}-E_{n_{1}, n_{2}, n_{3}}-i \hbar \Gamma_{0}} .
$$

Using expressions for the energy spectrum (3) and for one-particle wave functions (4), for the Green's function (7) we obtain:

$$
\begin{aligned}
& G\left(\mathbf{r}, \mathbf{R}_{a k} ; E_{\lambda}\right)=-(2 \pi)^{-\frac{3}{2}} \beta^{-\frac{1}{2}} a_{d}^{-3} \times \\
& \exp \left[-\frac{\left(x_{a k}-x_{0}\right)^{2}+y_{a k}^{2}+z_{a k}^{2}+\left(x-x_{0}\right)^{2}+y^{2}+z^{2}}{2 a^{2}}\right] \sum_{n_{1}=0}^{\infty}\left(\frac{1}{2}\right)^{n_{1}} \times \\
& \frac{H_{n_{1}}\left(\frac{x_{a k}-x_{0}}{a}\right) H_{n_{1}}\left(\frac{x-x_{0}}{a}\right)}{n_{1} !} \sum_{n_{2}=0}^{\infty}\left(\frac{1}{2}\right)^{n_{2}} \frac{H_{n_{2}}\left(\frac{y_{a k}}{a}\right) H_{n_{2}}\left(\frac{y}{a}\right)}{n_{2} !} \sum_{n_{3}=0}^{\infty}\left(\frac{1}{2}\right)^{n_{3}} \times \\
& \frac{H_{n_{3}}\left(\frac{z_{a k}}{a}\right) H_{n_{3}}\left(\frac{z}{a}\right)}{n_{2} !}\left(E_{\lambda}-\hbar \omega_{0}\left(n_{1}+n_{2}+n_{3}+\frac{2}{3}\right)+\frac{|e|^{2} E_{0}^{2}}{2 m^{*} \omega_{0}^{2}}-i \hbar \Gamma_{0}\right)^{-1} .
\end{aligned}
$$

Green's function (8) can be conveniently written in units of the effective Bohr radius $\left(a_{d}=4 \pi \epsilon_{0} \epsilon \hbar^{2} /\left(m^{*}|e|^{2}\right)\right.$, $\left(\epsilon_{0}\right.$ is the electric constant, $\epsilon$ is the static relative dielectric permeability of the QD) and the effective Bohr energy $E_{d}=\hbar^{2} /\left(2 m^{*} a_{d}^{2}\right)$. Let's use the obvious expression:

$$
\begin{gathered}
\left(E_{\lambda}-\hbar \omega_{0}\left(n_{1}+n_{2}+n_{3}+\frac{3}{2}\right)+\frac{|e|^{2} E_{0}^{2}}{2 m^{*} \omega_{0}^{2}}-i \hbar \Gamma_{0}\right)^{-1}= \\
E_{d}^{-1} \int_{0}^{+\infty} \exp \left(-E_{d}^{-1}\left(E_{\lambda}--\hbar \omega_{0}\left(n_{1}+n_{2}+n_{3}+\frac{3}{2}\right)+\frac{|e|^{2} E_{0}^{2}}{2 m^{*} \omega_{0}^{2}}+i \hbar \Gamma_{0}\right) t\right] d t= \\
E_{d}^{-1} \int_{0}^{+\infty} \exp \left[-\left(\epsilon_{q}+n_{1}+n_{2}+n_{3}\right) t\right] d t
\end{gathered}
$$

where $\epsilon_{q}=-\beta \eta_{q}^{2}+3 / 2-\beta W_{0}^{*}+i \Gamma_{0} \hbar \beta / E_{d} ; \eta_{q}^{2}=E_{\lambda} / E_{d} ; \beta=R_{0}^{*} /\left(4 \sqrt{U_{0}^{*}}\right) ; W_{0}^{*}=e^{2} E_{0}^{2} a_{d}^{2} \beta^{2} / E_{d} ; R_{0}^{*}=$ $2 R_{0} / a_{d} ; R_{0}$ is the QD radius; $U_{0}^{*}=U_{0} / E_{d}$; $U_{0}$ is the amplitude of the QD confinement potential, satisfying the relation $2 U_{0}=m^{*} \omega_{0}^{2} R_{0}^{2} ; \Gamma_{0}$ is the dissipative tunneling probability.

Then expression (8) can be represented as:

$$
\begin{aligned}
G\left(\mathbf{r}, \mathbf{R}_{a k} ; E_{\lambda}\right)=-(2 \pi)^{-\frac{3}{2}} \beta^{-\frac{1}{2}} E_{d}^{-1} a_{d}^{-3} \times \exp & {\left[-\frac{\left(x_{a k}-x_{0}\right)^{2}+y_{a k}^{2}+z_{a k}^{2}+\left(x-x_{0}\right)^{2}+y^{2}+z^{2}}{2 a^{2}}\right] \times } \\
\int_{0}^{\infty} d t \exp \left[-\varepsilon_{q} t\right] \times \sum_{n_{1}=0}^{\infty}\left(\frac{e^{-t}}{2}\right)^{n_{1}} & \frac{H_{n_{1}}\left(\frac{x_{a k}-x_{0}}{a}\right) H_{n_{1}}\left(\frac{x-x_{0}}{a}\right)}{n_{1} !} \sum_{n_{2}=0}^{\infty}\left(\frac{e^{-t}}{2}\right)^{n_{2}} \times \\
& \frac{H_{n_{2}}\left(\frac{y_{a k}}{a}\right) H_{n_{2}}\left(\frac{y}{a}\right)}{n_{2} !} \sum_{n_{3}=0}^{\infty}\left(\frac{e^{-t}}{2}\right)^{n_{3}} \frac{H_{n_{3}}\left(\frac{z_{a k}}{a}\right) H_{n_{3}}\left(\frac{z}{a}\right)}{n_{2} !} .
\end{aligned}
$$

Summation in (10) over quantum numbers $n_{1}, n_{2}, n_{3}$ can be performed using Mehler's formula [20]:

$$
\sum_{n=0}^{\infty}\left(\frac{e^{-t}}{2}\right)^{n} \frac{H_{n}\left(\frac{z_{a}}{a}\right) H_{n}\left(\frac{z}{a}\right)}{n !}=\frac{1}{\sqrt{1-e^{-2 t}}} \exp \left\{\frac{2 z_{a} z e^{-t}-\left(z_{a}^{2}+z^{2}\right) e^{-2 t}}{a^{2}\left(1-e^{-2 t}\right)}\right\} .
$$

As a result, for the Green's function, we have [17]:

$$
\begin{aligned}
G\left(\mathbf{r}, \mathbf{R}_{a k} ; E_{\lambda}\right) & =-(2 \pi)^{-\frac{3}{2}} \beta^{-\frac{1}{2}} E_{d}^{-1} a_{d}^{-3} \times \int_{0}^{\infty} d t \exp \left[-\varepsilon_{q} t\right]\left\{\left(1-e^{-2 t}\right)^{-\frac{3}{2}} \times\right. \\
& \left.\exp \left[-\frac{\left(\mathbf{r}-\mathbf{R}_{a k}\right)^{2}}{2 a^{2}} \operatorname{coth}(t)\right] \exp \left(-\frac{\left(x_{a k}-x_{0}\right)\left(x-x_{0}\right)+y_{a k} y+z_{a k} z}{a^{2}} \tanh \left(\frac{t}{2}\right)\right)\right\} .
\end{aligned}
$$


Using the procedure of the zero-range potential method, we obtain a dispersion equation that determines the dependence of the average binding energy of the resonant $\mathrm{g}$ - and u-states and the width of resonance levels on the coordinates for $D^{0}$-centers, parameters of the confinement potential of QDs, the strength of the external electric field, and parameters of dissipative tunneling. Applying the limits $\lim _{\mathbf{r} \rightarrow \mathbf{R}_{a k}}\left[1+\left(\mathbf{r}-\mathbf{R}_{a k}\right) \nabla_{r}\right]$ to both sides of expression (6), we obtain the following system of algebraic equations of the form [17]:

$$
\left\{\begin{array}{l}
c_{1}=\gamma_{1} a_{11} c_{1}+\gamma_{2} a_{12} c_{2} \\
c_{2}=\gamma_{1} a_{21} c_{1}+\gamma_{2} a_{2} 2 c_{2}
\end{array}\right.
$$

where $a_{k j}=\lim _{\mathbf{r} \rightarrow \mathbf{R}_{a k}}\left[1+\left(\mathbf{r}-\mathbf{R}_{a k}\right) \nabla_{r}\right] G\left(\mathbf{r}, \mathbf{R}_{a j} ; E_{\lambda}\right)(k, j=1,2)$.

Eliminating the coefficients $c_{i}$, containing the unknown wave function $\Psi_{\lambda}\left(\mathbf{r} ; \mathbf{R}_{a 1}, \mathbf{R}_{a 2}\right)$, from the system (13), we obtain the desired dispersion equation:

$$
\gamma_{1} a_{11}+\gamma_{2} a_{22}-1=\gamma_{1} \gamma_{2}\left(a_{11} a_{22}-a_{12} a_{21}\right)
$$

Let us find explicit expressions for the coefficients $a_{i i}$ and $a_{i j}$.

To isolate the diverging part in (12), we use the Weber integral [20]:

$$
\int_{0}^{+\infty} x^{-\frac{3}{2}} \exp \left[-\frac{\rho^{2}}{2 x}-\mu x\right] d x=\frac{\sqrt{2 \pi}}{|\rho|} \exp [-\sqrt{2 \mu}|\rho|], \quad\left[\Re\left(\rho^{2}\right)>0, \Re \mu>0\right]
$$

which in the notation adopted here has the form:

$$
\int_{0}^{\infty} t^{-\frac{3}{2}} d y \exp \left(-\varepsilon_{q} t\right) \exp \left(-\frac{\left|\mathbf{r}-\mathbf{R}_{a}\right|^{2}}{2 a^{2} t}\right)=\frac{\beta}{(2 \pi)^{2} \sqrt{\pi} E_{d} a_{d}^{2}} \frac{e^{-\sqrt{\varepsilon_{q}}\left|\mathbf{r}-\mathbf{R}_{a}\right|}}{\left|\mathbf{r}-\mathbf{R}_{a}\right|} .
$$

In this case, the Green's function can be represented as:

$$
\begin{aligned}
& G\left(\mathbf{r}, \mathbf{R}_{a k} ; E_{\lambda}\right)=-(2 \pi)^{-\frac{3}{2}} \beta^{-\frac{1}{2}} E_{d}^{-1} a_{d}^{-3} \times \int_{0}^{\infty} d t \exp \left[-\varepsilon_{q} t\right]\left\{\left(1-e^{-2 t}\right)^{-\frac{3}{2}} \times\right. \\
& \exp \left(-\frac{\left(x_{a k}-x_{0}\right)\left(x-x_{0}\right)+y_{a k} y+z_{a k} z}{a^{2}} \tanh \left(\frac{t}{2}\right)-\frac{\left(\mathbf{r}-\mathbf{R}_{a k}\right)^{2}}{2 a^{2}} \operatorname{coth}(t)\right)- \\
&\left.t^{-\frac{3}{2}} \exp \left[-\frac{\left(\mathbf{r}-\mathbf{R}_{a k}\right)^{2}}{2 a^{2} t} \operatorname{coth}(t)\right]\right\}-\frac{\beta}{(2 \pi)^{2} \sqrt{\pi} E_{d} a_{d}^{2}} \frac{\exp \left(-\sqrt{\varepsilon_{q}}\left|\mathbf{r}-\mathbf{R}_{a}\right|\right)}{\left|\mathbf{r}-\mathbf{R}_{a}\right|} .
\end{aligned}
$$

Again, applying the limits $\lim _{\mathbf{r} \rightarrow \mathbf{R}_{a k}}\left[1+\left(\mathbf{r}-\mathbf{R}_{a k}\right) \nabla_{r}\right]$ to both sides of this expression, we obtain [17]:

$$
\begin{aligned}
a_{k k}=-(2 \pi)^{-\frac{3}{2}} \beta^{-\frac{1}{2}} E_{d}^{-1} a_{d}^{-3}\left\{\int _ { 0 } ^ { + \infty } d t \operatorname { e x p } [ - \varepsilon _ { q } t ] \left(\left(1-e^{-2 t}\right)^{-\frac{3}{2}} \times\right.\right. \\
\left.\left.\exp \left[-\frac{\left(\left(x_{a k}-x_{0}\right)^{2}+y_{a k}^{2}+z_{a k}^{2}\right) \tanh (t / 2)}{a^{2}}\right]-(2 t)^{-\frac{3}{2}}\right)-\sqrt{\frac{\pi}{2}} \sqrt{\varepsilon_{q}}\right\}
\end{aligned}
$$


and

$$
\begin{aligned}
& a_{k j}=-(2 \pi)^{-\frac{3}{2}} \beta^{-\frac{1}{2}} E_{d}^{-1} a_{d}^{-3}\left\{\int _ { 0 } ^ { + \infty } d t \operatorname { e x p } [ - \varepsilon _ { q } t ] \left[\left(1-e^{-2 t}\right)^{-\frac{3}{2}} \times\right.\right. \\
& \exp \left[-\frac{\left(\left(x_{a k}-x_{a j}\right)^{2}+\left(y_{a k}-y_{a j}\right)^{2}+\left(z_{a k}-z_{a j}\right)^{2}\right) \operatorname{coth}(t)}{2 a^{2}}\right] \times \\
& \exp \left[-\frac{\left(x_{a k}-x_{0}\right)\left(x_{a j}-x_{0}\right)+y_{a k} y_{a j}+z_{a k} z_{a j}}{a^{2}} \tanh \left(\frac{t}{2}\right)\right]- \\
& \left.\left.t^{-\frac{3}{2}} \exp \left[-\frac{\left(\left(x_{a k}-x_{a j}\right)^{2}+\left(y_{a k}-y_{a j}\right)^{2}+\left(z_{a k}-z_{a j}\right)^{2}\right)}{2 a^{2} t} \operatorname{coth}(t)\right]\right]\right\}- \\
& \frac{\beta}{(2 \pi)^{2} \sqrt{\pi} E_{d} a_{d}^{2}}\left(\left(x_{a k}-x_{a j}\right)^{2}+\left(y_{a k}-y_{a j}\right)^{2}+\left(z_{a k}-z_{a j}\right)^{2}\right)^{-1} \times \\
& \exp \left(-\sqrt{\varepsilon_{q}\left(\left(x_{a k}-x_{a j}\right)^{2}+\left(y_{a k}-y_{a j}\right)^{2}+\left(z_{a k}-z_{a j}\right)^{2}\right)}\right) .
\end{aligned}
$$

In the case when $\gamma_{1}=\gamma_{2}=\gamma$ equation (14) splits into two equations that determine the symmetric (g-term) and antisymmetric (u-term) states of the electron, respectively, we obtain:

$$
\begin{gathered}
\gamma a_{11}+\gamma a_{12}=1, \quad\left(c_{1}=c_{2}\right) \\
\gamma a_{11}-\gamma a_{12}=1 . \quad\left(c_{1}=-c_{2}\right)
\end{gathered}
$$

In this case, the average binding energies of quasistationary g- and u-states are determined, respectively, as $\bar{E}_{g}=$ $E_{0,0,0}-\Re E_{2 \lambda u}, \bar{E}_{u}=E_{0,0,0}-\Re E_{2 \lambda g}$, and the broadening of impurity levels: $\Delta E_{g}=2 \Im E_{2 \lambda g}, \Delta E_{u}=2 \Im E_{2 \lambda u}$, respectively.

\section{Dependence of the average binding energies of quasistationary g- and u-states of the $D_{2}^{-}$-center and the width of impurity levels on the magnitude of the external electric field and parameters of dissipative tunneling.}

Figure 1 shows dependence of the average values of the binding energies for the quasistationary g-and u-states of the QD $D_{2}^{-}$-center on the magnitude of the external electric field and the parameters of 1D-dissipative tunneling, obtained by numerical analysis of equations (20) and (21). The field dependences of the binding energies average values for the quasistationary g- and u-states show dips that appear at the values of the parameters of 1D-dissipative tunneling and the external electric field strengths corresponding to the maxima in the field dependences of 1D-dissipative tunneling, with the participation.

Let us normalize the wave functions of quasistationary g- and u-states. From the normalization condition for the wave function $\Psi_{\lambda}\left(\mathbf{r} ; \mathbf{R}_{a 1}, \mathbf{R}_{a 2}\right)$, we have:

$$
\begin{array}{r}
\int_{V} d V\left|\Psi_{\lambda}\left(\mathbf{r} ; \mathbf{R}_{a 1}, \mathbf{R}_{a 2}\right)\right|^{2}=\gamma_{1}^{2} C_{1}^{2} \int_{-\infty}^{\infty} \int_{-\infty}^{\infty} \int_{-\infty}^{\infty}\left|G\left(\mathbf{r}, \mathbf{R}_{a 1} ; E_{\lambda}\right)\right|^{2} d x d y d z+ \\
2 \gamma_{1} \gamma_{2} C_{1} C_{2} \int_{-\infty}^{\infty} \int_{-\infty}^{\infty} \int_{-\infty}^{\infty} G\left(\mathbf{r}, \mathbf{R}_{a 1} ; E_{\lambda}\right) G\left(\mathbf{r}, \mathbf{R}_{a 2} ; E_{\lambda}\right) d x d y d z+ \\
\gamma_{2}^{2}\left|C_{2}^{2}\right|^{2} \int_{-\infty}^{\infty} \int_{-\infty}^{\infty}\left|G\left(\mathbf{r}, \mathbf{R}_{a 2} ; E_{\lambda}\right)\right|^{2} d x d y d z=1 .
\end{array}
$$

The integrals in expression (22) are calculated using the Green's function (7), i.e.: 


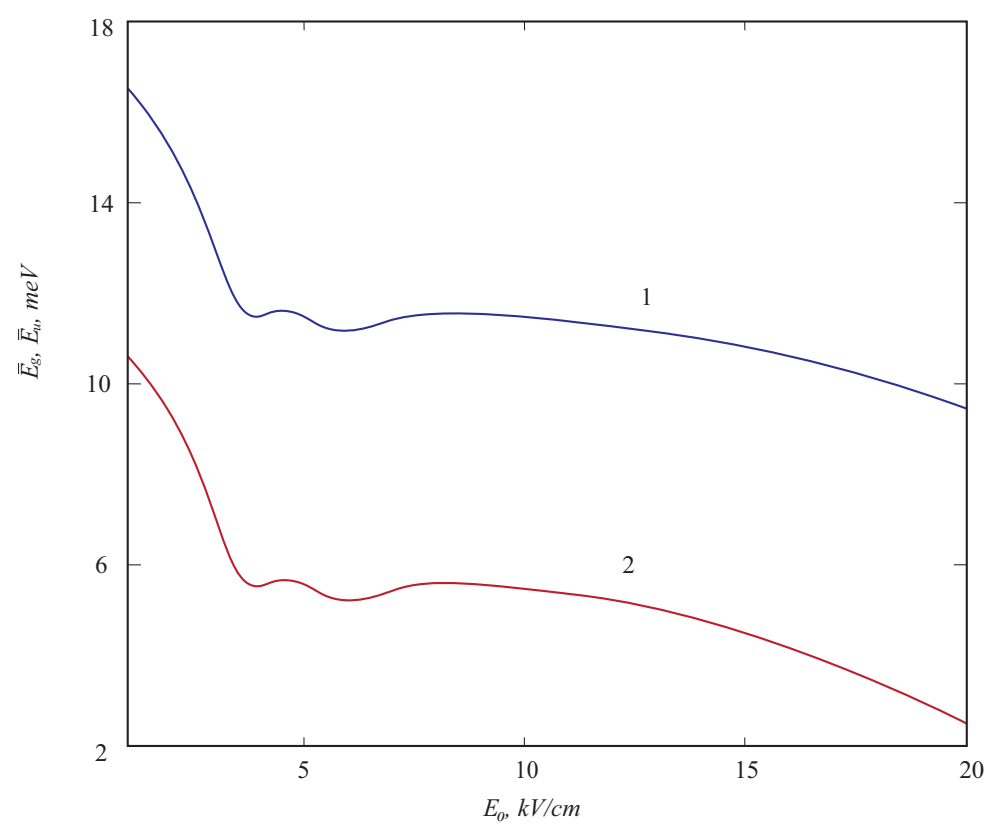

FIG. 1. Dependence of the average binding energy of quasistationary g-state $\bar{E}_{g}$ (curve 1 ), and ustate $\bar{E}_{u}$ (curve 2) for $D_{2}^{-}$-center on the strength of the external electric field $E_{0}$ in the presence of 1D-dissipative tunneling with allowance for the influence of two local phonon modes at $R_{0}=$ $50 \mathrm{~nm} ; U_{0}=0.35 \mathrm{eV} ; \eta_{i}=8.5 ; \rho_{12}=4.8 \mathrm{~nm} ; \epsilon_{T}^{*}=1.3 ; \epsilon_{L 1}^{*}=1.4 ; \epsilon_{L 2}^{*}=1.6 ; \epsilon_{C}^{*}=2.2$

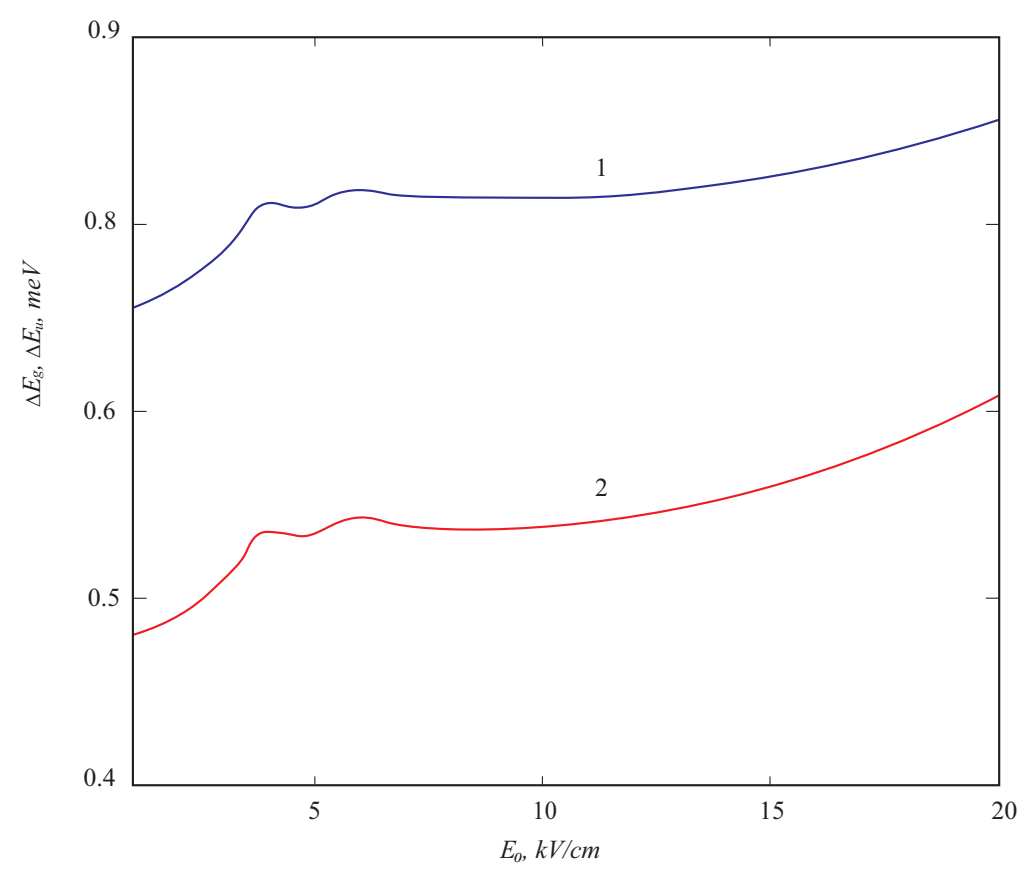

FIG. 2. Dependence of the broadening for impurity levels of quasistationary g- (curve 1), and ustates (curve 2) for $D_{2}^{-}$-center on the strength of an external electric field $E_{0}$ in the presence of 1Ddissipative tunneling taking into account the influence of two local phonon modes at $R_{0}=50 \mathrm{~nm}$; $U_{0}=0.35 \mathrm{eV} ; \eta_{i}=8.5 ; \rho_{12}=4.8 \mathrm{~nm} ; \epsilon_{T}^{*}=1.3 ; \epsilon_{L 1}^{*}=1.4 ; \epsilon_{L 2}^{*}=1.6 ; \epsilon_{C}^{*}=2.2$ 


$$
\begin{aligned}
& \int_{-\infty}^{\infty} \int_{-\infty}^{\infty} \int_{-\infty}^{\infty} G\left(\mathbf{r}, \mathbf{R}_{a j} ; E_{\lambda}\right) G\left(\mathbf{r}, \mathbf{R}_{a k} ; E_{\lambda}\right) d x d y d z= \\
& \sum_{n_{1}, n_{2}, n_{3}} \sum_{n_{1}^{\prime}, n_{2}^{\prime}, n_{3}^{\prime}} \frac{\Psi_{n_{1} n_{2} n_{3}}\left(\mathbf{R}_{a j}\right) \Psi_{n_{1}^{\prime}, n_{2}^{\prime}, n_{3}^{\prime}}^{*}\left(\mathbf{R}_{a k}\right)}{\left(E_{\lambda}-E_{n_{1}, n_{2}, n_{3}}\right)\left(E_{\lambda}-E_{n_{1}^{\prime}, n_{2}^{\prime}, n_{3}^{\prime}}\right)} \times \\
& \int_{-\infty}^{\infty} \int_{-\infty}^{\infty} \int_{-\infty}^{\infty} \Psi_{n_{1}, n_{2}, n_{3}}^{*}(\mathbf{r}) \Psi_{n_{1}^{\prime}, n_{2}^{\prime}, n_{3}^{\prime}}(\mathbf{r}) d x d y d z .
\end{aligned}
$$

We calculate the integral in (23) using the orthogonality condition for the eigenwave functions:

$$
\int_{-\infty}^{\infty} \int_{-\infty}^{\infty} \int_{-\infty}^{\infty} \Psi_{n_{1}, n_{2}, n_{3}}^{*}(\mathbf{r}) \Psi_{n_{1}^{\prime}, n_{2}^{\prime}, n_{3}^{\prime}}(\mathbf{r}) d x d y d z=\delta_{n_{1}, n_{1}^{\prime}} \times \delta_{n_{2}, n_{2}^{\prime}} \times \delta_{n_{3}, n_{3}^{\prime}}
$$

as a result, we have:

$$
\int_{-\infty}^{\infty} \int_{-\infty}^{\infty} \int_{-\infty}^{\infty} G\left(\mathbf{r}, \mathbf{R}_{a j} ; E_{\lambda}\right) G\left(\mathbf{r}, \mathbf{R}_{a k} ; E_{\lambda}\right) d x d y d z=\sum_{n_{1}, n_{2}, n_{3}} \frac{\Psi_{n_{1}, n_{2}, n_{3}}\left(\mathbf{R}_{a j}\right) \Psi_{n_{1}, n_{2}, n_{3}}^{*}\left(\mathbf{R}_{a k}\right)}{\left(E_{\lambda}-E_{n_{1}, n_{2}, n_{3}}\right)^{2}}
$$

where $j, k=1,2$.

The right-hand side of expression (25) can be written as:

$$
\sum_{n_{1}, n_{2}, n_{3}} \frac{\Psi_{n_{1}, n_{2}, n_{3}}\left(\mathbf{R}_{a j}\right) \Psi_{n_{1}, n_{2}, n_{3}}^{*}\left(\mathbf{R}_{a k}\right)}{\left(E_{\lambda}-E_{n_{1}, n_{2}, n_{3}}\right)^{2}}=-\frac{\partial G\left(\mathbf{R}_{a j}, \mathbf{R}_{a k} ; E_{\lambda}\right)}{\partial E_{\lambda}}=\left(\hbar \omega_{0}\right)^{-2} a^{-3} \frac{\partial G_{0}\left(\mathbf{R}_{a j}, \mathbf{R}_{a k} ; \varepsilon_{s}\right)}{\partial \varepsilon_{s}},
$$

where $G_{0}\left(\mathbf{r}, \mathbf{R}_{a} ; E_{\lambda}\right)$ - dimensionless Green's function.

Taking into account (23) - (26), we write down the normalization condition for the wave function $\Gamma_{\lambda}\left(\mathbf{r} ; \mathbf{R}_{a 1}, \mathbf{R}_{a 2}\right)$ of the quasistationary $D_{2}^{-}$-state:

$$
\begin{aligned}
\int_{V} d V\left|\Psi_{\lambda}\left(\mathbf{r} ; \mathbf{R}_{a 1}, \mathbf{R}_{a 2}\right)\right|^{2}= \\
\quad-\gamma^{2}\left(C_{1}^{2} \frac{\partial G\left(\mathbf{R}_{a 1}, \mathbf{R}_{a 1} ; E_{\lambda}\right)}{\partial E_{\lambda}}+2 C_{1} C_{2} \frac{\partial G\left(\mathbf{R}_{a 1}, \mathbf{R}_{a 2} ; E_{\lambda}\right)}{\partial E_{\lambda}}+C_{2}^{2} \frac{\partial G\left(\mathbf{R}_{a 2}, \mathbf{R}_{a 2} ; E_{\lambda}\right)}{\partial E_{\lambda}}\right)=1 .
\end{aligned}
$$

Then the expressions for the normalization factors of the symmetric $\left(C_{1}=C_{2}\right)$ and antisymmetric $\left(C_{1}=-C_{2}\right)$ $D_{2}^{-}$-states take the form:

$$
C_{1}^{2}=-\gamma^{2}\left\{\frac{\partial G\left(\mathbf{R}_{a 1}, \mathbf{R}_{a 2} ; E_{\lambda}\right)}{\partial E_{\lambda}} \pm 2 \frac{\partial G\left(\mathbf{R}_{a 1}, \mathbf{R}_{a 2} ; E_{\lambda}\right)}{\partial E_{\lambda}}+\frac{\partial G\left(\mathbf{R}_{a 2}, \mathbf{R}_{a 2} ; E_{\lambda}\right)}{\partial E_{\lambda}}\right\}^{-1},
$$

here the upper and lower signs refer to the g- and u-states, respectively. Let us calculate the derivatives in formula (28), passing to the dimensionless Green's function $G_{0}\left(\mathbf{r}, \mathbf{R}_{a} ; E_{\lambda}\right)$ by a simple transformation:

where

$$
G\left(\mathbf{r}, \mathbf{R}_{a} ; E_{\lambda}\right)=a^{-3}\left(\hbar \omega_{0}\right)^{-1} G_{0}\left(\mathbf{r}, \mathbf{R}_{a} ; \varepsilon_{q}\right)
$$

$$
G_{0}\left(\mathbf{r}, \mathbf{R}_{a} ; \varepsilon_{q}\right)=-2^{-1} \pi^{-\frac{3}{2}} \exp \left(-\frac{\left(x_{a}-x_{0}\right)\left(x-x_{0}\right)+y_{a} y+z_{a} z}{a^{2}}\right) \exp \left[-\frac{\left(\mathbf{r}-\mathbf{R}_{a}\right)^{2}}{2 a^{2}}\right] B\left(\frac{\varepsilon_{q}}{2},-\frac{1}{2}\right) .
$$

As a result, we have:

$$
\begin{aligned}
& \frac{\partial G_{0}\left(\mathbf{r}, \mathbf{R}_{a} ; \varepsilon_{q}\right)}{\partial \varepsilon_{q}}=-2^{-1} \pi^{-\frac{3}{2}} \exp \left(-\frac{\left(x_{a}-x_{0}\right)\left(x-x_{0}\right)+y_{a} y+z_{a} z}{a^{2}}\right) \frac{\partial B\left(\frac{\varepsilon_{q}}{2},-\frac{1}{2}\right)}{\partial \varepsilon_{q}}= \\
& 2^{-1} \pi^{-1} \exp \left(-\frac{\left(x_{a}-x_{0}\right)\left(x-x_{0}\right)+y_{a} y+z_{a} z}{a^{2}}\right) \frac{\Gamma\left(\frac{\varepsilon_{q}}{2}\right)}{\Gamma\left(\frac{\varepsilon_{q}}{2}-\frac{1}{2}\right)}\left(\psi\left(\frac{\varepsilon_{q}}{2}\right)-\psi\left(\frac{\varepsilon_{q}}{2}-\frac{1}{2}\right)\right),
\end{aligned}
$$


where $\psi(x)=\Gamma^{\prime}(x) / \Gamma(x)$ is the logarithmic derivative of the Euler gamma function $\Gamma(x)$.

Let us write down the final expression for the normalization factors of the wave functions $\Psi_{\lambda}\left(\mathbf{r} ; \mathbf{R}_{a 1}, \mathbf{R}_{a 2}\right)$ for the symmetric and antisymmetric states:

$$
\begin{aligned}
C_{1}=2^{\frac{5}{4}} \pi^{\frac{1}{2}} \beta^{-\frac{1}{4}} E_{d} a_{d}^{\frac{3}{2}} \gamma\left(\frac{\Gamma\left(\frac{\varepsilon_{q}}{2}\right)}{\Gamma\left(\frac{\varepsilon_{q}}{2}-\frac{1}{2}\right)}\left(\psi\left(\frac{\varepsilon_{q}}{2}\right)-\psi\left(\frac{\varepsilon_{q}}{2}-\frac{1}{2}\right)\right)\right)^{-\frac{1}{2}} \times \\
a^{2} \times \exp \left(-\frac{\left(x_{a 1}-x_{0}\right)^{2}+y_{a 1}^{2}+z_{a 1}^{2}}{a^{2}}\right) \pm 2 \exp \left(-\frac{\left(x_{a 1}-x_{0}\right)\left(x_{a 2}-x_{0}\right)+y_{a 1} y_{a 2}+z_{a 1} z_{a 2}}{a^{2}}\right)+ \\
\left.\exp \left(-\frac{\left(x_{a 2}-x_{0}\right)^{2}+y_{a 2}^{2}+z_{a 2}^{2}}{a^{2}}\right)\right\}^{-\frac{1}{2}} .
\end{aligned}
$$

Using expression (32) for the wave function of the quasi-stationary $D_{2}^{-}$- state in a QD in an external electric field, we obtain:

$$
\begin{aligned}
& \Psi_{\lambda}\left(\mathbf{r} ; \mathbf{R}_{a 1}, \mathbf{R}_{a 2}\right)=-2^{-\frac{1}{4}} \pi^{-1} \beta^{-\frac{1}{4}} a_{d}^{-\frac{3}{2}}\left(\frac{\Gamma\left(\frac{\varepsilon_{q}}{2}\right)\left(\psi\left(\frac{\varepsilon_{q}}{2}\right)-\psi\left(\frac{\varepsilon_{q}-1}{2}\right)\right)}{\Gamma\left(\frac{\varepsilon_{q}-1}{2}\right)}\right)^{-\frac{1}{2}} \times \\
& \left\{\exp \left(-\frac{\left(x_{a 1}-x_{0}\right)^{2}+y_{a 1}^{2}+z_{a 1}^{2}}{a^{2}}\right) \pm 2 \exp \left(-\frac{\left(x_{a 1}-x_{0}\right)\left(x_{a 2}-x_{0}\right)+y_{a 1} y_{a 2}+z_{a 1} z_{a 2}}{a^{2}}\right)+\right. \\
& \left.\exp \left(-\frac{\left(x_{a 2}-x_{0}\right)^{2}+y_{a 2}^{2}+z_{a 2}^{2}}{a^{2}}\right)\right\}^{-\frac{1}{2}} \times \\
& \left(\int_{0}^{\infty} d t \exp \left[-\varepsilon_{q} t\right](1-\exp (-2 t))^{-\frac{3}{2}} \exp \left(-\frac{\left(x-x_{a 1}\right)^{2}+\left(y-y_{a 1}\right)^{2}+\left(z-z_{a 1}\right)^{2}}{2 a^{2}} \operatorname{coth}(t)\right) \times\right. \\
& \exp \left(-\frac{\left(x_{a 1}-x_{0}\right)\left(x-x_{0}\right)+y_{a 1} y+z_{a 1} z}{a^{2}} \tanh \left(\frac{t}{2}\right)\right) \pm \int_{0}^{\infty} d t \exp \left[-\varepsilon_{q} t\right](1-\exp (-2 t))^{-\frac{3}{2}} \times \\
& \exp \left(-\frac{\left(x-x_{a 2}\right)^{2}+\left(y-y_{a 2}\right)^{2}+\left(z-z_{a 2}\right)^{2}}{2 a^{2}} \operatorname{coth}(t)\right) \times \\
& \left.\exp \left(-\frac{\left(x_{a 2}-x_{0}\right)\left(x-x_{0}\right)+y_{a 2} y+z_{a 2} z}{a^{2}} \tanh \left(\frac{t}{2}\right)\right)\right) .
\end{aligned}
$$

Here, the signs "+" and "-" determine the g- and u-states, respectively.

Expression (33) for the wave functions of quasistationary g- and u-states will make it possible to calculate the probabilities of radiative transitions of an electron in a quantum molecule in an external electric field.

\section{Conclusions}

In the model of the zero-range potential in the effective mass approximation, dispersion equations have been obtained that describe dependence of the average binding energies of the quasistationary g- and u-states of the $D_{2}^{-}$center in the QD, as well as the energy levels width on the magnitude of the external electric field and the parameters of 1D-dissipative tunneling. Dips in the field dependences of the average values of the binding energies of quasistationary g- and u-states have been revealed. The dips are caused by a significant decrease in the lifetime of the impurity quasi-stationary states at values of the dissipative tunneling parameters and external electric field strengths corresponding to the maxima on the field dependences of the 1D-dissipative tunneling probability. It is shown that the field dependences of the energy levels widths for the g- and u- states of the $D_{2}^{-}$-center have a resonance structure at the external electric field strengths corresponding to the dips in the field dependences of the average binding energies.

In the dipole approximation, the field dependence of the radiative transition probability (RTP) of an electron from a quasistationary u-state to a quasi-stationary g-state of the $D_{2}^{-}$-center in a QD in the presence of dissipative tunneling with the participation of two local phonon modes has been calculated. It was found that the curve of the RTP dependence on the strength of the external electric field contains three peaks. The leftmost peak corresponds to the RTP with the energy of the emitted photon comparable to the energy of the optical transition of an electron from 
the quasistationary u-state to the quasi-stationary g-state of the $D_{2}^{-}$-center. The other two peaks are separated by a dip and are due to the presence of two local phonon modes; moreover, with a decrease in the phonon frequency, the peaks are smoothed out and the dip is transformed into a horizontal section, the length of which substantially depends on the constant of interaction with the contact medium.

\section{Acknowledgements}

This work was supported by the Ministry of Education and Science of the Russian Federation (project 0748-20200012) and RFBR (project no. 18-42-130007 p_a). The research was partially supported by the Foundation for Polish Science through the IRA Programme co-financed by EU within SG OP.

\section{References}

[1] Aleshkin V.Y., Gavrilenko L.V., Odnoblyudov M.A., Yassievich I.N. Impurity resonance states in semiconductors. Semiconductors, 2008, 42, P. 880-904.

[2] Ovchinnikov A.A., Dakhnovsky Yu.I., et al. Principles of controlled modulation of low-dimensional structures. UNC DO (a publishing company at Moscow State University), Moscow, 2003, 510 p.

[3] Leggett A.J. (Ed.) Controllable dissipative tunneling. Tunnel transport in low-dimensional systems. Fizmatlit, Moscow, 2012 , 495 p.

[4] Mantsevich V.N. Nonequilibrium effects and non-stationary electron transport in semiconductor nanostructures with interparticle interaction. PhD Thesis, Moscow, 2014, 337 p.

[5] Mantsevich V.N., Maslova N.S. Spatial effects of Fano resonance in local tunneling conductivity in vicinity of impurity on semiconductor surface. JETP Lett., 2010, 91, P. 139-142.

[6] Maltezopoulos T., Bolz A., et al. Wave-function mapping of InAs quantum dots by scanning tunneling spectroscopy. Phys. Rev. Lett., 2003, 91, 196804.

[7] Tserkovnyak Y., Halperin B.I., Auslaender O.M., Yacoby A. Finite-size effects in tunneling between parallel quantum wires. Phys. Rev. Lett., 2002, 89, 136805.

[8] Bomze Yu., Mebrahtu H., et al. Resonant tunneling in a dissipative environment. Phys. Rev. B, 2009, 79, 241402.

[9] Qin H., Holleitner A.W., Eberl K., Blick R.H. Coherent superposition of photon- and phonon-assisted tunneling in coupled quantum dots. Phys. Rev. B, 2001, 64, 241302.

[10] Trocha P., Rudziński W. Phonon-assisted electronic transport through double quantum dot system coupled to ferromagnetic leads. Acta Physica Polonica A, 2013, 124, P. 843-845.

[11] Liu L., Du Y., Zhou H., Lin T. Resonant tunneling through a coupled double quantum well in the presence of electron-phonon interaction. Phys. Rev. B, 1996, 54, P. 1953-1958.

[12] Kang K., Min B.I. Effect of quantum confinement on electron tunneling through a quantum dot. Phys. Rev. B, 1997, 55, P. 15412-15415.

[13] Kusmartsev F.V., Krevchik V.D., et al. Phonon assisted resonant tunneling and its phonons control. JETP Lett., 2016,104, P. $392-397$.

[14] Polyakov A.M. Gauge Fields and Strings. Routledge, London, 1987. 312 p.

[15] Coleman S. Aspects of Symmetry: Selected Erice Lectures. Cambridge University Press, Cambridge, 1985,402 p.

[16] Benderskii V.A., Vetoshkin E.V , Kats E.I. Semiclassical quantization of bound and quasistationary states beyond the adiabatic approximation. Phys. Rev. A, 2004, 69, 062508.

[17] Zhukovsky V.Ch., Krevchik V.D., et al. The effect of an external electric field on the optical properties of a quantum-dot molecule with a resonant state of the $D_{2}^{-}$center. Moscow University Physics Bulletin, 2013, 68, P. 397-404.

[18] Baz' A.I., Zeldovich Ya.B., Perelomov A.M. Scattering, Reactions and Decays in Nonrelativistic Quantum Mechanics. Jerusalem: Israel Program for Scientific Translations, 1969, $246 \mathrm{p}$.

[19] Galitski V., Karnakov B., Kogan V., Galitski V. (Jr). Exploring Quantum Mechanics: A Collection of 700+ Solved Problems for Students, Lecturers, and Researchers. Oxford University Press, Oxford, 2013, 904 p.

[20] Bateman H. Higher Transcendental Functions [Vol. 1-3]. McGraw-Hill Book Company, New York, 1953. 